\title{
ALIMENTACIÓN, NUTRICIÓN Y DIETÉTICA A TRAVES DE LA HISTORIA DE LA ENFERMERÍA
}

\author{
Natividad SÁnchez González. Profesora Titular de Fundamentos de Enfermería. \\ María José García Meseguer. Profesora Titular de Farmacología Clínica y Dietética. \\ Universidad de Castilla - La Mancha. Escuela Universitaria de Enfermería de Albacete.
}

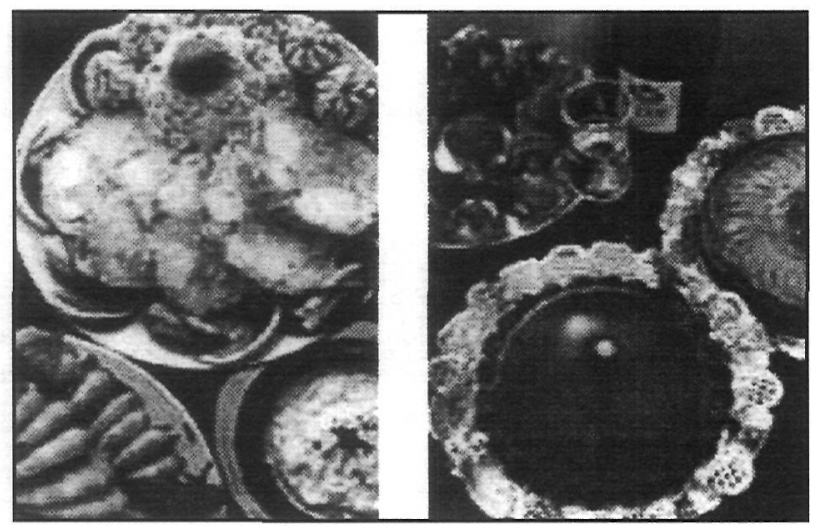

\section{RESUMEN}

Este trabajo muestra una síntesis de la presencia de la alimentación como cuidado básico en la atención de Enfermería desde la Edad Media en que aparecen los primeros documentos escritos sobre cuidados realizados por una persona concreta, donde se pone de manifiesto la importancia de los diferentes alimentos en la atención de personas tanto sanas como enfermas.

\section{FOOD, NUTRITION AND DIETETICS THROUGH NURSING HISTORY}

\section{SUMMARY}

The present work shows that nutrition has been considered a basic care in nursing since the Middle Ages. There is plenty of written evidence of the benefits received by a person who is provided with the care offered by an adequate diet, be they either healthy or sick.

\section{INTRODUCCIÓN}

Este análisis de la relación entre Enfermería, alimentación, nutrición y dietética lo iniciamos a partir de la Edad Media, periodo en el que se han encontrado documentos escritos referentes a los cuidados y necesidades de individuos sanos y enfermos realizados por personas con esta función concreta. Dichos documentos proceden en su mayoría de hospitales (en estas instituciones era preceptivo llevar un registro de gastos), y su contenido proporciona una valiosa información sobre la institución y su entorno.

Analizaremos de forma cronológica algunos ejemplos de los estudios realizados a través de dichos documentos.

\section{SIGLO XIII}

Un estudio de Hernandez y Pinar (1997), tiene como fuente un INCUNABLE (textos impresos que recogen el saber popular en cuanto a remedios para enfermedades genéricas y orientaciones sobre medidas higiénicas), impreso en 1494, Propietatibus Rerum ( de las propiedades de las cosas), autor Fray Bartolomé Anglico. Este ejemplar se encuentra en el Instituto Histórico Dominicano de Salamanca.

De esta obra se extraen desde la óptica de Enfermería importantes elementos de cuidados básicos entre los que se incluye la alimentación. Según las autoras "pueden ser considerados en algunas de sus secciones como una guía para la salud y una educación en los autocuidados, temas hoy de 
absoluta actualidad y que constituyen la base de la Enfermería en atención primaria, salvaguardando las distancias.

Esta obra dedica dos capítulos a la alimentación y las autoras han clasificado su contenido en los siete puntos siguientes :

1.- Definición y clasificación: Se identifica la "vianda" como lo que hoy entendemos por alimentación. Respecto a la bebida hace una clasificación que atiende a las propiedades del líquido injerido, distinguiendo entre agua (como elemento indispensable y sin a porte calórico), líquidos ( con contenido calórico, como el vino, los caldos) y otras bebidas con propiedades medicinales (arropes, pócimas e incluso aguas con propiedades específicas).

2.- Ingesta, absorción y eliminación : Explica el procesado del alimento desde su ingesta hasta su asimilación por el organismo y su destino final; Siempre en la línea de los conocimientos de la época y en un vocabulario llano.

3.- Consumo energético y reposición: Relación actividad física - ingesta. Relaciona la necesidad de reposición según las circunstancias y edades.

4.- Propiedades organolécticas: El autor ateniéndose al saber de la época, profundiza en aquellas características que están estudiadas y son más evidentes para la población, como la textura y la densidad de los alimentos y la calidad, el olor y el sabor de los vinos.

5.- Contenido calórico: Tiene conocimientos sobre el diferente aporte calórico de los alimentos e incluso, tiene la consideración de valorar las diferentes épocas del año como diversificadoras en contenido calórico.

6.- "Cultura alimentaria" de la época: En algunos aspectos presenta grandes diferencias con la actual como el desaconsejar las verduras crudas y las frutas poco maduras. En otros, no difiere mucho de la cultura popular actual, por ejemplo, aconseja tomar agua caliente en ayunas si se ha comido o bebido mucho la noche anterior, como purgante.

7.- La alimentación en el sano y en el enfermo: Las autoras consideran un ejemplo de los que hoy podrían ser consejos de Enfermería, en la distinción que Fray Bartolomé Anglico hace sobre la alimen- tación y la bebida diferenciadas e individualizadas en el sano y en el enfermo, incluso especificándose la adecuación según la enfermedad que se padezca.

Hernández y Pinar terminan su análisis concluyendo que, " extrapolando situaciones, estos consejos los compararíamos a las recomendaciones elementales que se divulgan en pequeños folletos desde los diversos organismos públicos, dirigidos a la población general". Su fin es orientar y educar en los hábitos alimentarios conducentes a la salud.

\section{SIGLO XIV}

En el estudio realizado por Tey i Freixa (1996), referido al Hospital D`en Colón (Barcelona 1.375), encontramos un clara vinculación de la alimentación a la Enfermería. Su análisis se basa en un manuscrito conservado en el Archivo Capitular de la Catedral de Barcelona que lleva por título Capbreu del spital d’ em Colom ( relación de bienes del Hospital de Colón ).

Siguiendo este análisis en el apartado referente a la atención de necesidades de los enfermos y los sanos, la autora refleja que, " si bien la información disponible no permite esbozar siquiera un régimen alimenticio propio para cada uno de los estamentos de la institución (sanos, niños y enfermos), ofrece una muestra suficientemente variada de alimentos como para poder afirmar que los mínimos nutricionales estaban asegurados".

En sus conclusiones puntualiza que las necesidades de nutrición de los acogidos en el hospital se atendían tan bien como los recursos de la institución y las costumbres de la época lo permitían: En conjunto, escasa aportación de proteínas, probable exceso de grasa, pobreza de lácteos, y en consecuencia escaso aporte de ciertos minerales y carencia de vitaminas durante el invierno.

Todo esto no es muy distinto de lo que era habitual en aquella sociedad, salvo si se trataba de mesas opulentas en las cuales también eran notables los desequilibrios pese a su abundancia.

\section{SIGLO XV}

De finales de este siglo exponemos, como ejemplo, el estudio realizado sobre el Hospital del Rey de Burgos por Santo Tomás y cols. (1996), a través del libro de visitas del monasterio de Las Huelgas y 
sus filiaciones en que fue visitador D. Fernando Vázquez de Arce prior de la Iglesia de Osma en tiempos de los Reyes Católicos, año de 1.500, documentos que, en la actualidad, se encuentra en el Monasterio de El Escorial. De esta fuente las autoras del análisis desprenden, entre otras, las siguientes conclusiones respecto a la relación entre la administración de cuidados y la alimentación:

Había enfermeras y mujeres seglares ( que no se denominaban enfermeras), que se dedicaban de forma específica al cuidado de los enfermos día y noche. La alimentación constituía un elemento importante de estos cuidados mientras que otros como la higiene no son tenidos en cuenta.

En cuanto a la tipología de los cuidados, la alimentación era el principal elemento para cuidar la salud del cuerpo, lo que se deduce por la compra de alimentos que se realizaba en el Hospital para el consumo exclusivo de los enfermos y por la descripción de los utensilios de que disponían las enfermeras para este menester.

Este largo periodo de mil años, la Edad Media, da paso a una etapa en la que comienza una evolución general del conocimiento y que por tanto, también afecta al tema que estamos tratando.

\section{SIGLO XVI}

Encontramos dos muestras de los que pudo ser el cuidado de la alimentación (dentro de instituciones hospitalarias), por los trabajos llevados a cabo por Delgado Marchante (1996), respecto a la situación en el Hospital de Laborantes de San Lorenzo de El Escorial, y por Hernández y Pinar (1996) en cuanto a la Enfermería en lo Hospitales Madrileños de este siglo.

El primero de ellos se fundamenta, entre otros documentos, en la obra de Zarco Cuevas (1923), quien transcribió las Instrucciones de este Hospital, uno de los primeros creados en España para atender a los accidentados en los trabajos de construcción del Real Monasterio. Del trabajo de Delgado se desprende la importancia de la alimentación en el cuidado de los enfermos, tanto en lo horarios de comidas como en el tipo de alimentos según la enfermedad.

La segunda aportación caracteriza la Enfermería en los hospitales de Madrid en un difí- cil periodo en el que se intenta la concentración y secularización hospitalaria y en el que se esboza una finalidad más curativa de la institución. Hernández y Pinar en su trabajo describen el quehacer diario de la Enfermería y anotan entre los cuidados básicos la alimentación, donde destacamos las siguientes características:

- La alimentación es uno de los cuidados básicos del enfermo y las dietas quedan al albedrío de los médicos.

- El hecho de que aparezcan muchas "recetas", de elaboración de los alimentos alude a la importancia que adquiere la dieta dentro de la curación en esta etapa y, probablemente, a la escasez de principios básicos en la alimentación.

- Se trata de adecuar, cosa que parece lógica, el tipo de alimentación en cada época y para cada enfermo.

\section{SIGLO XVII}

Continuando nuestro orden cronológico, hemos extraído como ejemplo para reflejar la vinculación de la Enfermería al cuidado de la alimentación del enfermo, el estudio realizado por Fernández Mérida (1996), sobre la aportación de la Orden de S. Juan de Dios en Málaga durante la epidemia de peste acaecida en Este siglo, concretamente 1.637. La principal fuente bibliográfica han sido las Actas Capitulares del Archivo municipal.

Siguiendo a esta autora, desde la perspectiva de la historia de la Enfermería, resulta de gran interés abordar las intervenciones desarrolladas en períodos epidémicos ya que en estas situaciones es cuando se recoge más información de carácter sanitario, revelando las necesidades y carencias que se dan en el campo asistencial. Así, figura dentro del tratamiento de la peste una especial atención a la alimentación e hidratación, aconsejando la provisión de hidratos de carbono, proteínas y líquidos de preferencia ácidos, y desaconsejando alimentos contaminantes o que se injieran crudos. También recoge normas básicas en la alimentación, como buena presentación comer despacio o beber poco durante la comida.

La primera publicación hasta ahora conocida sobre el oficio de enfermero pese a carecer de regulación legislativa, data de 1.617 . Se trata de la obre de 
Fray Andrés Fernández, Hermano Obregón, Instrucción de enfermeros para aplicar los remedios a todo género de enfermedades y acudir a muchos accidentes que sobrevienen en ausencia de médicos.

En dicha obra se hace referencia al cuidado básico de alimentación especificando los principios que se han de seguir según el tipo de enfermos. También se considera el agua un elemento importante en la regulación de la temperatura corporal y la hidratación .

\section{SIGLO XVIII}

Como en cualquier disciplina y en cualquier época, la mayoría de la bibliografía surge de una necesidad que la sociedad demanda sobre diferentes aspectos para solucionar, al menos de forma parcial, un problema. Así el s. XVIII constituyó un periodo crucial y de avance para la futura profesionalización de la Enfermería .

Tomando como referencia el estudio realizado por Sánchez, Ortega y Elbal (1996), se hace referencia al texto que con el título, "Nuevo thesoro de medicina, sacado de los aphorísmos de la caridad, según la práctica de muchos enfermeros capuchinos, así españoles como italianos", fue editado en 1.750 siendo su autor Fray Gil de Villalón enfermero capuchino. En este texto, dedicado a la instrucción de los enfermeros se dedican dos capítulos al cuidado básico de la alimentación, explicando con todo detalle los alimentos que deben administrarse a los enfermos según las patologías.

\section{SIGLOS XIX Y XX}

En los dos últimos siglos de este milenio se ha producido por un lado, el reconocimiento legal y académico de la Enfermería como profesión, lo que ha permitido su consolidación como disciplina y una rápida evolución, y por otro, desde la perspectiva de la Nutrición, el desarrollo de ésta como Ciencia.

La aplicación de los conocimientos científicos de la Nutrición a uno de los cuidados considerados como básicos desde el punto de vista de la Enfermería, la alimentación, requiere del apoyo de la Dietética, lo que significa un avance paralelo entre estas tres disciplinas y una necesaria conjunción de las mismas.
Por tanto la disposición de equipos de profesionales de la salud adecuadamente formados en el ámbito de Nutrición y Dietética contribuirá de forma muy notable a la disminución de la incidencia de malnutrición hospitalaria, disminución de factores de riesgo cardiovascular y a una mejor información y educación tanto para el individuo sano como para el enfermo sobre el tipo de alimentación más adecuada a sus necesidades y requerimientos.

\section{BIBLIOGRAFÍA}

Delgado Marchante, A. (1996) El hospital de laborantes de San Lorenzo de el Escorial. Actas I Congreso Nacional de Enfermería, Barcelona: 17-21.

Hernández Martín, F. y Pinar García, ME (1996) La enfermería en los hospitales madrileños del siglo XVI. Características generales del hospital de la Edad Media. Actas I Congreso Nacional de Historia de la Enfermería. Fundación Uriach, Barcelona: 43-51.

Hernández Martín, F. y Pinar García, ME. (1997) Acerca de la salud. Orientaciones para una vida saludable en un texto del siglo XIII. Separata del Archivo Dominicano, T. XVIII, Salamanca.

Tey y Freixá, R. (1996) Una aproximación a la enfermería medieval. El Hospital D`em Colom en el año 1375. Actas I Congreso Nacional de Historia de la Enfermería. Fundación Uriach, Barcelona: 33-38.

Santo Tomás, M; Castro, E; Castanedo, C; Fernández, ML y Melchor, A. (1996) Alimentación en la Baja Edad Media. Base de los cuidados. Rol, 214: 55-57.

Sánchez, N; Ortega, C y Elbal, L. (1996)Historia de la enfermería a través de las instituciones de Castilla-La Mancha. Colegio de Enfermería de Albacete, Albacete.

Zarco Cuevas, (1923) Publicaciones sobre el monasterio de El Escorial. Revista Ciudad de Dios: 132-134.

Fernández Mérida, MC. (1996) Málaga y la epidemia de peste de 1637. Aportación de la orden de San Juan de Dios. Actas I Congreso Nacional de Historia de la Enfermería. Fundación Uriach, Barcelona: 25-28. 